\title{
II CONFERÊNCIA DE POLÍTICAS PÚBLICAS PARA \\ MULHERES: RELATÓRIO DA II CONFERÊNCIA \\ MUNICIPAL DE POLÍTICAS PARA AS MULHERES DO \\ MUNICÍPIO DE RIO CLARO - II CMPM
}

Izabel Cristina REZENDE ${ }^{1}$

\begin{abstract}
RESUMO
O objetivo deste breve artigo é expor os principais resultados da II Conferência de Políticas Públicas para Mulheres para a reflexão e adoção de políticas públicas para as mulheres em Rio Claro. As políticas públicas se constroem a partir do cotidiano das mulheres e a II Conferência Municipal demonstra a importância das mulheres em pautar o governo e a sociedade, sendo fundamental dar voz as mulheres conforme o seu território e na sua diversidade. Claro que vamos precisar de muitas conferências e encontros para diminuir a desigualdade de gênero e raça no Brasil, mas vamos em frente reafirmando a participação das mulheres na construção de uma sociedade justa.
\end{abstract}

Palavras-chave: Políticas Públicas. Direitos das Mulheres. Rio Claro.

\section{CONFERENCE ON PUBLIC POLICIES FOR WOMEN: REPORT OF THE II MUNICIPAL CONFERENCE ON POLICIES FOR WOMEN IN THE MUNICIPALITY OF RIO CLARO - II CMPM}

\begin{abstract}
the objective of this brief paper is to expose the main results of the II Women's Public Policy Conference for reflection and adoption of public policies for women in Rio Claro. As public policies are constructed from the daily lives of women and the II Municipal Conference demonstrates the importance of women in guiding the government and society, is essential to give women a voice according to their territory and in their diversity. Of course, we will need many conferences and meetings to reduce gender and race inequality in Brazil, but we go ahead reaffirming women's participationin building a just society.
\end{abstract}

Keywords: Public Policy. Women's Rights. Rio Claro.

\footnotetext{
${ }^{1}$ Assistente Social. Assessora Municipal de Referência e Atendimento à Mulher de Rio Claro/SP. E-mail: bellrezendeartesanato@gmail.com
} 


\title{
II CONFERENCIA SOBRE POLÍTICAS PÚBLICAS PARA MUJERES: INFORME DE LA II CONFERENCIA MUNICIPAL SOBRE POLÍTICAS PARA LA MUJER EN EL MUNICIPIO DE RIO CLARO - II CMPM
}

\begin{abstract}
RESUMEN
el propósito de este breve artículo es mostrar los principales resultados de la II Conferencia de Políticas Públicas para Mujeres para la reflexión y la adopción de políticas públicas para la mujer en Rio Claro. Las políticas públicas se basan en la vida cotidiana de las mujeres y la II Conferencia Municipal demuestra la importancia de las mujeres para guiar al gobierno y la sociedad, siendo esencial darles una voz de acuerdo con su territorio y diversidad. Por supuesto, necesitaremos muchas entrevistas y reuniones para reducir la desigualdad de género y raza en Brasil, pero reafirmaremos la participación de las mujeres en la construcción de una sociedad justa.
\end{abstract}

Palabras clave: Políticas públicas. Derechos de las mujeres. Rio Claro.

\section{INTRODUÇÃO}

Motivada pelas discussões tidas em minha participação na mesa de encerramento do II Congresso Brasileiro de Organização do Espaço intitulada "Marielle Franco: Democracia, Direitos Humanos e resistência popular no Brasil", exponho este artigo que tem como objetivo, apresentar os principais resultados da Conferencia para a reflexão e adoção de políticas públicas para as mulheres em Rio Claro.

A II Conferência Municipal de Políticas para as Mulheres, com o tema: "Mais Direitos, Participação e Poder para as Mulheres", foi realizada no dia 26/09/2015 na rua 6 nº3265 NAM - Núcleo Administrativo Municipal - Alto do Santana no município de Rio Claro/SP. A mesma foi organizada pela Conferência Municipal, sendo realizada como etapa eletiva e chamada pelo Poder Executivo.

O objetivo da realização da ll Conferência Municipal de Políticas Públicas para as Mulheres é a construção da igualdade tendo como perspectiva o fortalecimento da autonomia econômica, cultural religiosa e política das mulheres, contribuindo para erradicação da extrema pobreza e para o exercício da cidadania das mulheres no Brasil considerando a diversidade. 
O artigo está organizado em cinco partes, sendo a primeira está introdução. A segunda apresenta as considerações gerais do evento em questão. $\mathrm{Na}$ terceira temos os resultados obtidos no evento. Na quarta parte são apresentados os eixos que canalizaram as discussões e resultaram nas principais decisões e recomendações da Conferência. Na quinta é feita a conclusão deste texto.

\section{RELATÓRIO DAS DELIBERAÇÕES DA II CONFERÊNCIA MUNICIPAL DE POLÍTICAS PARA MULHERES DE RIO CLARO (SP)}

A II Conferência Municipal de Políticas para as Mulheres foi realizada sábado, 26, no Auditório do Núcleo Administrativo Municipal (Rua 06, 3265, Alto do Santana) das 12:00 às 17:00 horas e teve a participação de aproximadamente 150 pessoas, que escolheram oito delegadas para representarem Rio Claro na etapa estadual, em novembro de 2015.

A Comissão Organizadora da II CMPM foi composta por representantes do poder público e sociedade civil, que são: Izabel Cristina Rezende da Assessoria Municipal de Referência e Atendimento à Mulher, Kizie de Paula Aguiar da Assessoria Municipal de Integração Racial, Marta Barbosa Ceccato da Secretaria Municipal de Assistência Social, Divanilde de Paula do Conselho Municipal dos Direitos da Mulher, Ana Claudia Silva do Coletivo de Juventude Negra "Nekenge" e Aline Luiz Camargo do Conselho Municipal dos Direitos da Mulher. Tivemos a participação de 83 pessoas, sendo 33 pessoas do poder público, representados pelos seguintes órgãos: Fundação Municipal de Saúde, Câmara Municipal de Piracicaba, Câmara Municipal de Rio Claro, Secretaria Municipal de Assistência Social, Gabinete do Prefeito de Rio Claro, Delegacia de Rio Claro, Secretaria Municipal de Governo, Universidade Estadual Paulista "Júlio de Mesquita Filho" (UNESP- Campus Rio Claro), OAB- Comissão da Mulher Advogada, Conselho Tutelar e 50 pessoas da sociedade civil representando entidades, Sindicato dos Químicos, Coletivo Feminista MariaMaria, Terreiro de Umbanda Lucia Oiá e Caboclo 7 Cachoeiras. 
A conferência analisou a situação atual, estabeleceu diretrizes e indicou caminhos para atingir os desejos e as necessidades expressas pela população, em especial, as mulheres.

Durante a mesa de abertura, a vice-prefeita Olga Salomão (Partido dos Trabalhadores - PT) destacou a "disposição das mulheres rio-clarenses de discutirem suas questões e observou que os assuntos tratados são prioritários na agenda de uma sociedade que quer ser mais participativa e igualitária”.

Os participantes se reuniram em quatro grupos para discutirem os eixos temáticos centrais da conferência. O Grupo I avaliou a contribuição dos conselhos de direitos da mulher e dos movimentos feministas na efetivação da igualdade de direitos e oportunidades para as mulheres, em sua diversidade e especificidades: avanços e desafios; o Grupo II avaliou as estruturas institucionais e políticas desenvolvidas para as mulheres nos âmbitos municipal, estadual e federal; o Grupo III discutiu recomendações para reforma política com inclusão das mulheres; e o Grupo IV avaliou o Sistema Nacional de Políticas para as Mulheres: subsídios e recomendações.

Participaram do evento a presidente do Fundo Social de Solidariedade, Rosana Pinhatti Altimari; a vereadora Raquel Picelli (PT); a presidente do Conselho Municipal dos Direitos da Mulher, Guaraci de Paula Pereira; a assessora de Referência e Atendimento à Mulher, Izabel Rezende (autora deste artigo); a Presidente do Conselho Municipal da Comunidade Negra, Divanilde Aparecida de Paula; a diretora do Sistema Único de Assistência Social, Sônia Maria Catel Gerner; a representante do Movimento LGBT de Rio Claro, Yanca Pimentel; e Silvana do Amaral Verissimo, representando o Conselho Nacional dos Direitos da Mulher.

Foram eleitas as seguintes delegadas: Izabel Rezende, Kizie de Paula e Rosecler Santos, como representantes do governo municipal, e Divanilde Aparecida de Paula (Movimento de Mulheres Negras), Ana Claudia Guedes (Coletivo Feminista de Rio Claro - Maria Maria), Cassiana Rodrigues de Oliveira Dias (Movimento Sindical), Yanca Pimentel (Movimento LGBT/Mulher Trans) e Araci Leme Soares (Mulher de Terreiro) como representantes da sociedade civil. 
O evento foi organizado pela Diretoria de Políticas Especiais - Assessoria de Referência e Atendimento à Mulher, no qual sua gestora presidiu a Conferência, em parceria com o Conselho Municipal dos Direitos da Mulher juntamente com a Presidente do mesmo.

Como parte da programação da conferência, a palestra magna ficou por conta da representante do Conselho Nacional dos Direitos da Mulher, a Senhora Silvana Veríssimo, que foi indicada pela Ministra das Mulheres, da Igualdade Racial e dos Direitos Humanos Eleonora Menicucci.

A Conselheira Silvana Veríssimo fez uma palestra sobre a participação ativa das mulheres e empoderamento. Palestra que enriqueceu o debate, pois veio no sentido de capacitar todas/os participantes da plenária, já que o tema central da sua fala foi sobre a construção da democracia e da cidadania com a justa participação das mulheres. A palestra foi interativa e os participantes se sentiram à vontade para somar junto a palestrante.

\section{RESULTADOS DA CONFERÊNCIA}

A II Conferência Municipal de Políticas para Mulheres foi realizada em parceria com a Assessoria de Referência e Atendimento à Mulher e do Conselho Municipal dos Direitos da Mulher, juntamente com uma equipe de apoio de alunas da UNESP, Conselheira do Conselho dos Direitos da Mulher e representante da Secretaria de Planejamento e Meio Ambiente, além da comissão organizadora.

Foram realizadas 18 pré-conferências em toda cidade e também nos territórios, tais como: Igreja Evangélica, Comunidades tradicionais - Terreiro de Umbanda, Grupo de Dolas, mulheres de escola de samba, setorial de mulheres do Partido dos Trabalhadores, Associações de Bairros, Projeto Social (que tem como objetivo resgatar autoestima de meninas e meninos em situação de vulnerabilidade) e Mulheres Negras. Sendo assim, foram ouvidas, aproximadamente, 460 mulheres na sua diversidade e especificidade para realmente avaliar se as políticas para mulheres estão sendo aplicada na transversalidade.

Dentre os temas discutidos, destaco a flexibilidade das reuniões do Conselho dos Direitos da Mulher, buscando a participação das mulheres 
trabalhadoras, pois só assim elas teriam condições de fazer discussões com segurança. Na verdade, precisamos divulgar mais o papel do CMDM.

Na cidade de Rio claro, temas como Plano Municipal de Educação que foi aprovado no município com algumas ressalvas, nos grupos de trabalho ficou claro quando as mães dizem que é preciso capacitar os professores pois cada criança é diferente uma das outras e devem ser tratadas com igualdade. As mulheres também falaram do enfrentamento à violência, uma vez que com a reengenharia do governo Estadual, a Delegacia dos Direitos da Mulher, ficou centralizada com as demais delegacias dificultando o acesso da mulher vitimada e que as leis devem ser mais duras com os homens, em cumprimento à Lei Maria da Penha.

O que causou impacto nesta conferência, em reação à primeira, é que as mulheres conhecem seus direitos, estão buscando autonomia, mas quando chega na parte da política, elas deixam por último a participação da Mulher no espaço de poder, tudo se torna empecilho. Assim, ainda existe a necessidade de irmos a campo para falar da Reforma política.

Acredito que a mulher, ocupando espaço de poder, é fundamental para garantirmos a diversidade e a implementação de políticas públicas.

Diante dos temas trabalhados, verificou-se a necessidade de implantar um Sistema Nacional de Atendimento à Mulher, nos moldes do SUAS ou SUS, para a garantia de seus direitos.

\section{EIXO TEMÁTICOS DA II CONFERENCIA MUNICIPAL}

Os quatro eixos da II Conferencia Municipal de Rio Claro resultaram na identificação de desafios, avanços e recomendações que foram aprovadas, como podemos observar a seguir.

O eixo I "Contribuição dos Conselhos dos Direitos da mulher e de Movimentos Feministas e de Mulheres para a efetivação da igualdade de direito e oportunidade para as Mulheres em sua diversidade e especificidades” identificou os seguintes desafios e avanços: 


\section{Desafios:}

1 - Que o Conselho Municipal dos Direitos da Mulher tenha um fundo de repasse financeiro para capacitação, divulgação de campanhas e biblioteca, propiciando o fortalecimento do mesmo;

2 - Que o Conselho da Mulher faça parceria com as escolas e empresas, para fortalecer a discussão de gênero focando nos homens, com o objetivo de diminuir a desigualdade de gênero;

3 - Que o Conselho Municipal dos Direitos da Mulher faça maior divulgação em todos os meios de comunicação, através do rádio, jornal, ônibus e folder, esclarecendo a população da existência do mesmo e de suas finalidades e enfatizando dias e horários de reuniões. Garantir a elaboração da cartilha municipal da Mulher, que constam todas as políticas públicas do município, o papel do conselho e como se dá a participação. Assim como, a realização de reuniões itinerantes e em horários das $14 \mathrm{hs}$ às $16 \mathrm{hs}$ e/ou alternados em espaços públicos e em territórios do CRAS.

Avanços

1 - Revisão da Lei que institui o Conselho Municipal dos Direitos da Mulher tendo em vista as transformações da realidade local como Mulheres Trans, Lésbicas, Bissexuais...

2- Que a secretaria de Segurança Pública garanta a participação de uma representante nas reuniões do Conselho Municipal dos Direitos da Mulher;

3- Que sejam mais bem utilizados os espaços públicos já existentes, para ampliar as atividades voltadas à Mulher.

O segundo eixo, por sua vez, com o tema "Estruturas Institucionais $e$ políticas públicas desenvolvidas para Mulheres no âmbito municipal, estadual e federal":

Desafios:

1 - Que o Estado garanta a efetivação da DDM - Delegacia da Mulher 24hs, garantindo o funcionamento nos finais de semana e feriados, sendo equipada e com equipe capacitada para o atendimento humanizado, com estruturas adequadas e com atendimento de assessoria jurídica e psicológica em cumprimento da lei Maria da Penha. 
2 - Criação da Casa Abrigo Regional para Mulheres vítimas de violência doméstica, respeitando a diversidade e suas especificidades, aproveitando o espaço físico de prédios ociosos do município.

3 - Agilidade nas consultas e nos resultados de exames de alta complexibilidade ginecológicos, ressonância magnética, oftalmologia, ultrassom transvaginal e endovaginal e mamografia. Que o SUS tenha um serviço de informatização para evitar desvio de resultados.

Os avanços propostos no segundo eixo são os seguintes:

1 - Garantir campanha e mutirões relacionados à saúde da Mulher em todas as faixas etárias, com orientação do uso de métodos anticoncepcionais, as DST, gravidez;

2 - Que o poder Judiciário crie a Vara da Família;

3 - Garantir mais prevenção na questão da violência na família, como descentralização em parceria com o CREAS e CRAM, juntamente com as empresas privadas e públicas.

Já no eixo III "Sistema político com participação das Mulheres e Igualdade" foram feitas duas recomendações:

1 - Urgente efetivação do Pacto de Enfrentamento à Violência contra a Mulher do Estado com o Município;

2 - Garantir no mínimo CINQUENTA POR CENTO de Mulheres no espaço político, e que, nos partidos políticos, que se respeite a diversidade nas três esferas de poder - Legislativo, Executivo e Judiciário, inclusive nas nomeações de secretarias nas instâncias. Ainda, recomendase que haja capacitação das mulheres para que sejam empoderadas para assumir estes espaços políticos.

Por fim, uma recomendação é feita no eixo IV "Sistema Nacional de Política para as Mulheres: Subsídios e Recomendações”: criação de um Sistema Único Nacional de Políticas para Mulheres com dotação orçamentária, informatizado, capacitado, transversal, dividido em básica, média e alta complexidade e com flexibilidade para adequação de cada município. 


\section{CONSIDERAÇÕES FINAIS}

As políticas públicas se constroem a partir do cotidiano das mulheres e a II Conferência Municipal demonstra a importância das mulheres em pautar o governo e a sociedade, sendo fundamental dar voz as mulheres conforme o seu território e na sua diversidade.

Claro que vamos precisar de muitas conferências e encontros para diminuir a desigualdade de gênero e raça no Brasil, mas vamos em frente reafirmando a participação das mulheres na construção de uma sociedade justa. 
ANEXOS

Figura 1 - Cartaz de divulgação de pré-Conferência

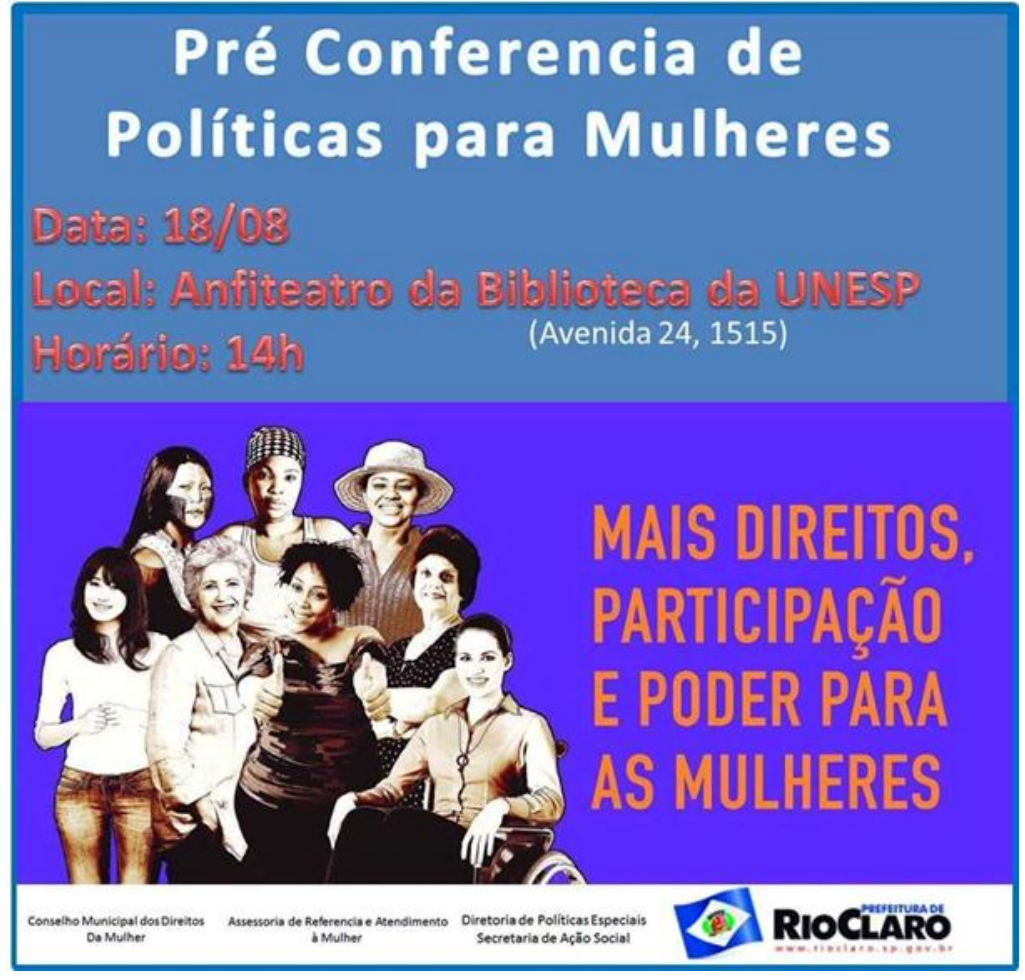

Figura 2 - Cartaz de divulgação de pré-Conferência

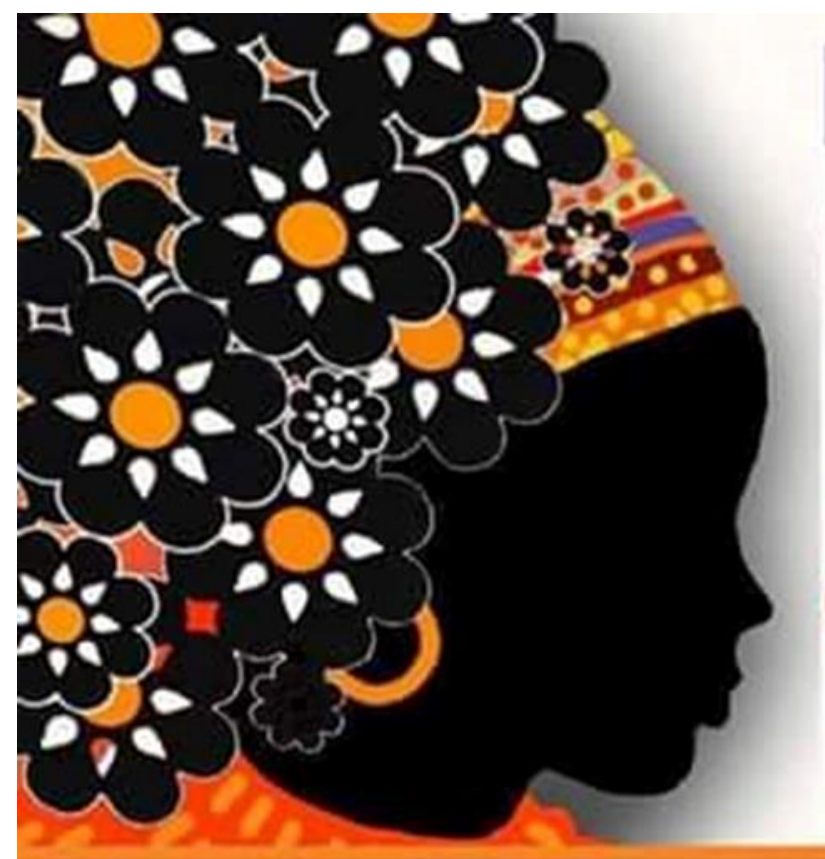

Pré Conferencia de Politicas para Mulher

PRÉ das PRETAS

\section{Quando? 29/08 Onde? Diretoria de Politicas Especiais Av. atio Claro of Av 26} Horário? 15h 

de Políticas Públicas para as Mulheres no município de Rio Claro - II CMPM

Figura 3 - Cartaz de divulgação de pré-Conferência

\section{PRÉ CONFERENCIA DE POLÍTICAS PARA MULHERES}

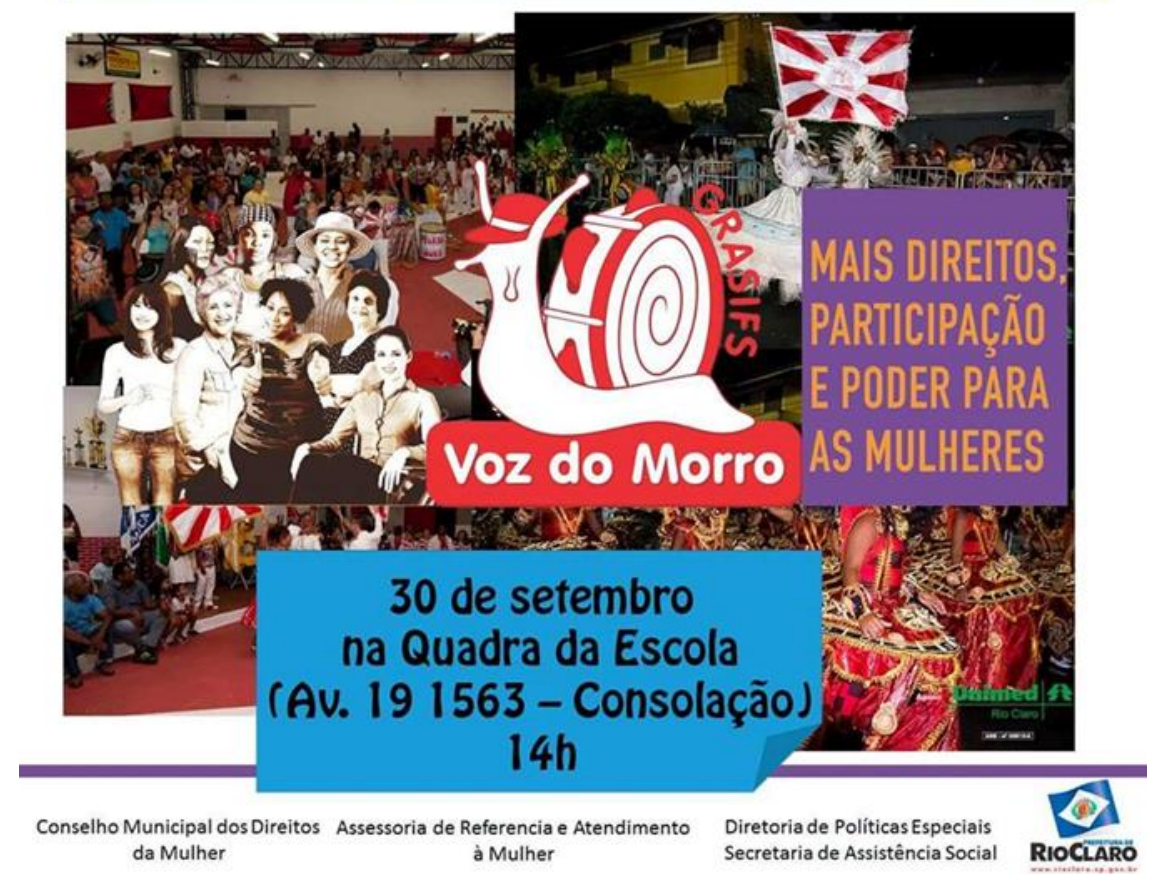

Figura 4 - Cartaz de divulgação da II Conferência

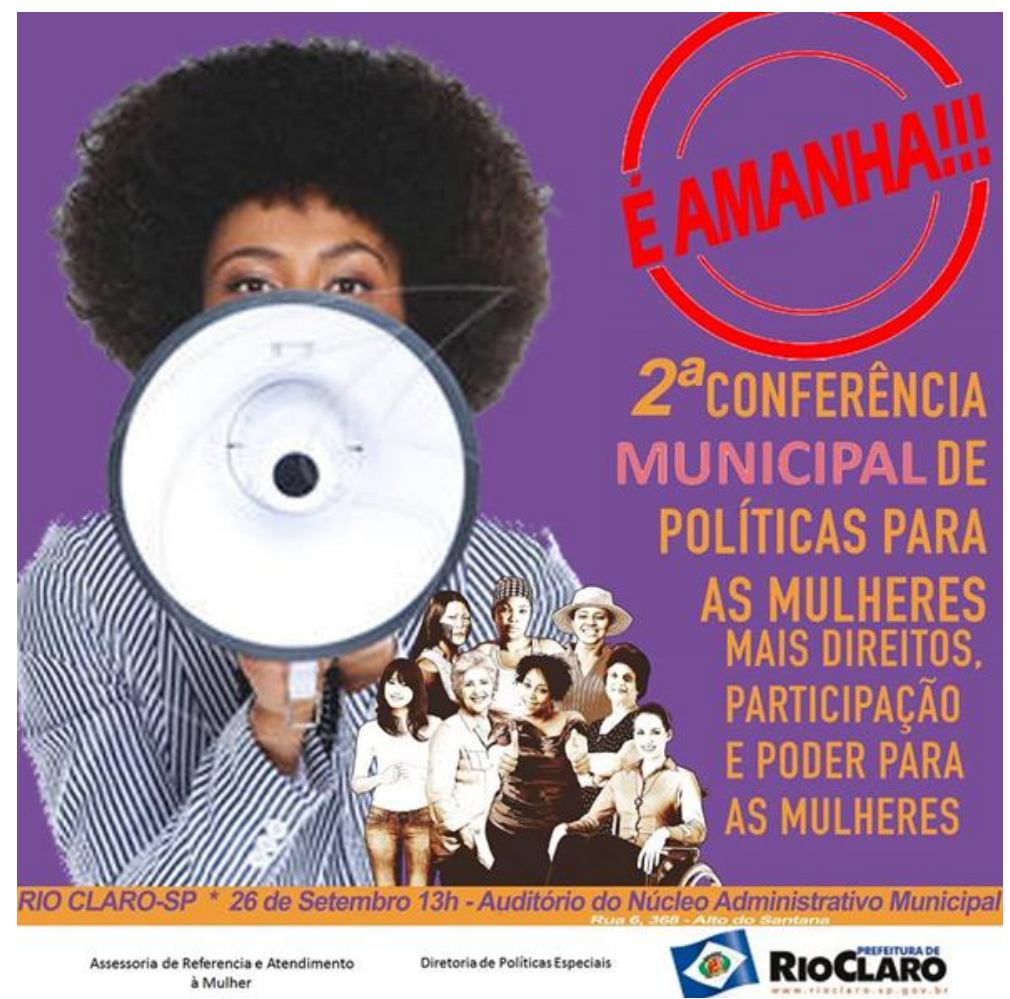


Figura 5 - Equipe organizadora da II Conferência de Políticas Públicas para Mulheres

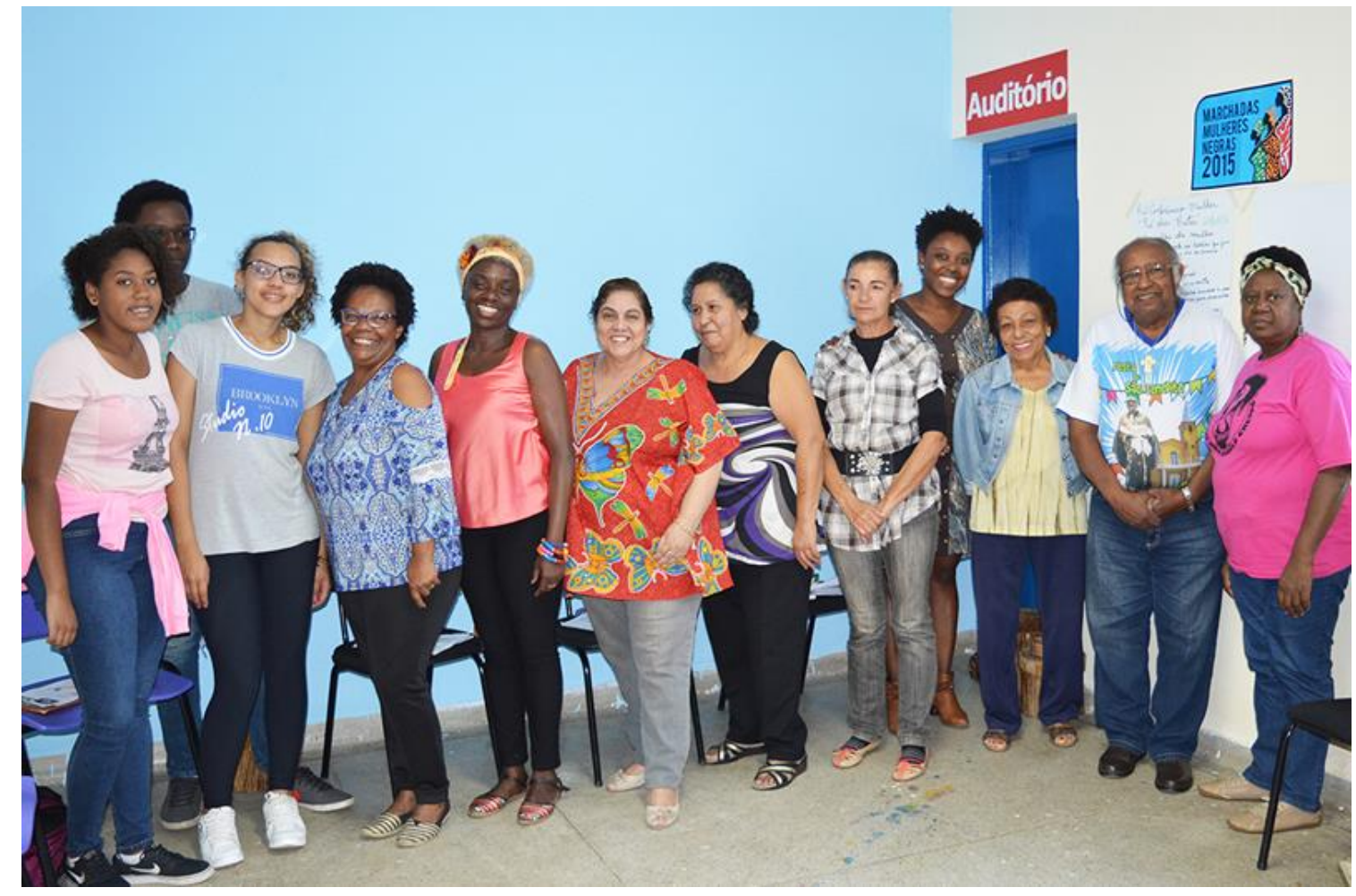

